\title{
An Introduction to Practical Laboratory Optics, by J. F. James
}

\section{Giovanni Volpe}

To cite this article: Giovanni Volpe (2015) An Introduction to Practical Laboratory Optics, by J. F. James, Contemporary Physics, 56:4, 493-495, DOI: 10.1080/00107514.2015.1074618

To link to this article: https://doi.org/10.1080/00107514.2015.1074618

$$
\text { Published online: } 11 \text { Aug } 2015 .
$$

Submit your article to this journal $₫$

Llll Article views: 89

View Crossmark data ¿ 


\title{
ESSAY REVIEW
}

\section{An Introduction to Practical Laboratory Optics}

\author{
Giovanni Volpe \\ Soft Matter Lab, Physics Department, Bilkent University, Ankara, Turkey
}

A review of An Introduction to Practical Laboratory Optics, by J. F. James, Cambridge, Cambridge University Press, 2014, 187 pp., $£ 33.89$ (paperback), ISBN 978-1-107-68793-6. Scope: monograph, manual, guide. Level: early career researcher, researcher, specialist.

Science and technology have been transforming our lives at an ever more rapid pace over the last few centuries and, in particular, over the last few decades. This unprecedented rate of progress has been intimately entangled to the development of ever more powerful scientific instruments, which have paved the way to the exploration of the extremely small and the unthinkably big. Often, these instruments are in fact optical instruments, relying on the most advanced optical techniques.

Simple lenses have been in use since ancient times to correct simple vision defects such as myopia and presbyopia. However, it was only during late sixteenth century that the first compound microscope appeared in the Netherlands, most likely an invention of eyeglass makers. During the seventeenth century, the interest in microscopic techniques grew at a steady pace, attracting the attention of scientists in Italy, such as Galileo Galilei, and in England, such as Robert Hooke, as well as in the Netherlands, where the greatest contribution came from Antonie van Leeuwenhoek. The latter developed the microscope and applied it to the study of biological problems, discovering in the process red blood cells and spermatozoa and, in 1676, reporting the first observation of micro-organisms. From there, microscopic techniques have grown fast during the nineteenth and twentieth centuries in parallel with an ever-deeper understanding of the nature and behaviour of light. In 1881, Ernst Abbe discovered how to produce diffraction-limited images. Taking advantage of the recently invented electric illumination, in August 1893, Köhler developed his namesake illumination, which is still in use in modern microscopes. In 1953, Frits Zernike introduced the phase contrast method to illuminate a sample and, in 1955, George Normarski introduced the differential phase contrast method. In the last decades, even more powerful techniques have been developed. These techniques permit one to focus light far below the diffraction limit down to nanometric spots, for example, making use of near-field techniques and plasmon resonances. Furthermore, even more powerful technique have been developed in order to manipulate small objects - such as colloidal particles, cells and molecules - using the force that can be exerted by a focused light beam, a technique known as optical tweezers.

In parallel to the development of optical instruments to explore the extremely small, there has been a development also of optical instruments to explore the unthinkably big. The first undisputed claims of having built a telescope go back to the beginning of the seventeenth century in the Netherlands, again the work of eyeglass makers. These first Dutch telescopes were composed of a convex lens and of a concave lens. This design permitted them to magnify the image several times without inverting it; therefore, these telescopes were mainly used for terrestrial observations, for example, at sea or in a battlefield. Galileo Galilei, while working at the University of Padua in Italy, improved and perfected the technique employed to build telescopes. Using the telescopes he built, Galileo Galilei performed his famous discoveries of the satellites of Jupiter, of the spots on the sun, of the phases of Venus and of the hills and valleys on the Moon. Incidentally, the observation of the revolution of the satellites of Jupiter around its planet gave Galileo Galilei the confidence to support the Copernican system against the Ptolemaic system, getting him into troubles with the Catholic Church of Rome. The next big step forward in the development of telescopes came with the invention of reflective telescopes, which make use of reflecting mirrors instead of lenses in order to focus the image. These were the telescopes that Isaac Newton favoured, as, based on his theory of light and colours, he expected them to be free from chromatic aberrations. The further development of telescopes continued in the following centuries mainly for astronomical observations, leading to the great telescopes currently in use both on Earth and in space.

Of course, microscopes and telescopes are not the only optical instruments to have been developed. On the one hand, the instruments we have described so far make use only of a small portion of the light spectrum, corresponding typically to visible and near-infrared light; nevertheless interesting and important discoveries have 
been done using instruments capable of harnessing other regions of the spectrum, from X-rays to radio waves. On the other hand, there are also optical instruments designed not just for imaging application. For example, spectrometers permit one to separate the various wavelengths composing a light beam and have been widely employed for the characterisation of materials, and interferometers have been used to study the wave nature of light.

Given the importance of optical instruments, a book that explains how to build them and teaches the tricks of the trade has a clear place in the bookshelf of anyone interested in experimental optics. James' An Introduction to Practical Laboratory Optics. is just this kind of book. This is a valuable contribution as it goes down to the practicalities and tricks involved in the building of an optical set-up. It teaches a lot of tips and tricks, which, by personal experience, one normally only learns after many years in the trade of building optical instruments and only if one has the luck to have an appropriate guidance. Appropriately, while some historical notes are provided, mostly the book goes straight down to the point of teaching the basic knowledge needed to understand and build optical set-ups.

In fact, even though we employ optics in our daily life, often we do so without understanding its principles and, therefore, in a sub-optimal way. James gives a striking example at the very beginning of the book:

The most famous and illustrious mis-user of an optical instrument is Sherlock Holmes. There is an iconic figure of him with Inverness cape, deerstalker hat and calabash pipe, peering through a magnifying glass, the latter held at harm's length, to inspect a possible blood strain.

While it is nice to see that the most famous investigator uses science - and, in particular, optics - in his crime scene investigation, he does it wrong. And this image, printed on the cover of most Sherlock Holmes novels, can be credited to have misinformed and misled multitudes of children, including myself some years ago, trying to put to a good use a magnifying glass. James readily corrects Sherlock Holmes:

This is the wrong way to use a magnifying glass. [...] The glass should be held close to the eye [...] and the object brought in until it is in clear focus at a comfortable distance for viewing.

Thus, with this book, James sets out to teach us how to build and operate an optical instrument correctly.

The first chapter of the book is dedicated to the introduction of some basic concepts related to the two most common optical elements: lenses and mirrors. The author introduces the basic concepts of ray of light and optical path, and then he proceeds to define other concepts such as numerical aperture, étendue and vignette. The second chapter focuses on the various schemes that have been proposed to build optical telescopes and binoculars, describing a wide range of telescope designs, from the original Galilean telescopes to the Keplerian refractors and the Newtonian reflectors. Chapter 3 deals with the eyepieces that are used especially in handheld devices, such as binoculars, and in microscopes. Even though James pays always attention to give some historical background and context, these first three chapters are quite schematic and dry, and might have benefitted from some more detailed illustrations.

Chapters 4 and 5 deal with cameras and objectives. Typically, in optical laboratories, cameras and objectives are bought from commercial manufactures. In consideration of this fact, the author goes to some details in explaining how these devices work and in describing their properties. I found these two chapters useful as they provide enough background information - information that is needed whenever making a decision on what elements to buy. Of course, one drawback of any discussion regarding scientific cameras nowadays is the fast pace of progress in this field, which means that in some years the material explained in Chapter 5 might be outdated - but maybe by then it might be time for a second edition of the book.

Spectrometry is dealt with in Chapter 6. The discussion is quite thorough, as is appropriate for such an important family of optical instruments. It starts with the discussion of a spectroscope based on a simple prism mounted on a goniometer. This is the most classical design of a spectrometer invented by Robert Bunsen and Gustav Kirchhoff in the early nineteenth century. The author takes advantage of the discussion of this classical instrument to introduce some important concepts such as the resolving power and the efficiency of a spectrometer. Afterwards, the author moves to more complex and modern designs, such as the ones that make use of diffraction gratings and monochromators. I was particularly pleased to see that the author discussed in detail how to align from scratch a spectrometer and the effects of potential disturbances and misalignments that might arise because of thermal effects. It is not too surprising the detail and proficiency with which the author describes the implementation and use of spectrometers, as this topic is within his most direct field of expertise and, in fact, he had published a previous book dedicated to the design of spectrographs (James, Spectrograph Design Fundamentals, Cambridge University Press, 2011).

Another very important optical instrument is the interferometer, to which Chapter 7 is dedicated. The discussion is detailed, even though less personal than the one of the spectrometer in Chapter 6. The author first describes the basic working principles and design parameters of a generic interferometer and then discusses all classical interferometer designs including the Fizeau, 
Michelson, Fabry-Pérot, Mach-Zehnder and Sagnac interferometers. Unfortunately, only a small section, corresponding to less than a third of a page, is dedicated to optical resonators; given the importance of coherent light sources in current science and technology, this topic would certainly have deserved a more in-depth discussion and it definitively deserves a dedicated chapter in a possible second edition of the book.

Chapter 8 is dedicated to the description of polarised light and of its use and manipulation in the laboratory. This is quite a short chapter, which introduces the necessary basic concepts without too many details.

Chapter 9 discusses very briefly the implementation of microscopes with a short paragraph on Köhler illumination. Probably, this is the chapter that I found most missing the essential information. In fact, illumination in microscopy is a very important field and in any practical application of optics, the optimisation of the illumination is quite crucial and difficult. I would have liked a more in-depth discussion of various illumination techniques, including a more detailed discussion of how Köhler illumination can be implemented step by step. Similarly, I also found lacking Chapter 10 on siderostats and coelostats and Chapter 11 on the detection and measurement of radiation. Reading the book one has a strong impression the author got tired after Chapter 8 and decided to wrap up the book without too many details. This is a pity, and an issue to be addressed in a possible second edition of the book.
The final chapter deals with some very practical aspects of the work of an experimental optical scientist such as how (and when) to clean and store lenses and mirrors, how to mount optical components, and how to understand when and how to use optical filters. Again, this chapter is quite short and could have been a bit more comprehensive, but I nevertheless found it extremely useful, especially for beginners, as it explicitly states some basic knowledge that is needed whenever working on building an optical instrument, but is often not explicitly stated.

In conclusion, this book is a practical and no-nonsense guide to the implementation of optical instruments with a focus on telescopes, spectroscopes, interferometers and microscopes. It is a good starting point for those starting to work on experimental optics and it can also be useful to experienced practitioners such as myself - while reading this book, I learnt lots of tricks and pieces of information I did not know beforehand. Obviously, this is nevertheless a small introductory book, where many important topics are not covered. In particular, the implementation of resonant cavities to generate coherent laser light and the use of optical fibres are missing.

Giovanni Volpe

Soft Matter Lab, Physics Department, Bilkent University Ankara, Turkey giovanni.volpe@fen.bilkent.edu.tr (C) 2015, Giovanni Volpe 